\title{
Topology Preserving Brain Tissue Segmentation Using Graph Cuts
}

\author{
Xinyang Liu ${ }^{1}$, Pierre-Louis Bazin ${ }^{2}$, Aaron Carass ${ }^{3}$, and Jerry Prince ${ }^{3}$ \\ ${ }^{1}$ Brigham and Women's Hospital, Boston, MA \\ 1 xinyangebwh.harvard.edu \\ ${ }^{2}$ MPI for Human Cognitive and Brain Sciences, Leipzig, Germany \\ $2_{\text {bazinecbs.mpg.de }}$ \\ ${ }^{3}$ Johns Hopkins University, Baltimore, MD \\ ${ }^{3}$ \{aaron_carass, prince\}@jhu.edu
}

\begin{abstract}
In segmentation of magnetic resonance brain images, it is important to maintain topology of the segmented structures. In this work, we present a framework to segment multiple objects in a brain image while preserving the topology of each object as given in an initial topological template. The framework combines the advantages of digital topology and several existing techniques in graph cuts segmentation. The proposed technique can handle any given topology and enforces object-level relationships with little constraint over the geometry. We apply our algorithm to brain tissue segmentation and demonstrate its accuracy and computational efficiency.
\end{abstract}

\section{Introduction}

In neuroimaging, topology is particularly important for the study of the cerebral cortex; with a few changes to the anatomy, one can assume that the cortex has the topology of a hollow sphere. From this assumption, the highly convoluted surface of the brain can be flattened into a plane, a sphere or a partially flattened surface and structural or functional information can be visualized and analyzed more easily. Topology is also an important issue for computational anatomy based on diffeomorphisms, for statistical shape models using parametric models, and for physical simulations of flow or tissues.

Bazin and Pham proposed a technique, known as topology-preserving anatomy-driven segmentation (TOADS) [2], which respects the topology of structures during the segmentation of magnetic resonance brain images. They further improved the method by incorporating topology of groups of objects and statistical atlases [3], to better segment subcortical structures with similar intensities. Combining fast marching and intensity-based clustering algorithms, the TOADS framework guarantees strict topological equivalence between the segmented image and an initial topological template, and is therefore more robust to noise compared with other tissue classification methods which only use intensity information. Although TOADS is superior in many aspects, its computation is relatively time consuming. More efficient topology preserving segmentation method would be favorable.

Based on the max-flow/min-cut theory, graph cuts is an efficient combinatorial energy minimization tool and has become popular in the past decade to solve vision problems such as image restoration, stereo and segmentation [5]. For the segmentation of binary image (with only foreground and background), it has the advantage of fast computation, global optimization and low sensitivity to initialization. Graph cuts was first introduced by Greig et al. [12], and was enhanced when Boykov and Kolmogorov proposed an efficient max-flow/min-cut algorithm [5] and further applied to N-d image segmentation by Boykov and Funka-lea [4]. Since one drawback of graph cuts is its inherent binary optimization, many works have focused on applying graph cuts to multi-label problems, e.g., in [11, 14, 18]. Among them the most influential two are $\alpha$ expansion and $\alpha-\beta$ swap, which are two fast approximation algorithms of global energy optimum, proposed by Boykov et al. [6]. To take topology constraints into consideration, Zeng et al. proposed a topology preserving segmentation algorithm [19] for binary images called topology cuts, based on the aforementioned Boykov-Kolmogorov algorithm. The technique was further simplified and validated by Danek and Maska [10]. Hower et al. is the first to apply graph cuts to 3-D brain image segmentation [14], however, they did not address topological 
issues.

In this work, we developed a framework to segment multiple objects in an image while preserving the topology of each object as given in an initial topological template. The framework combines $\alpha-\beta$ swap to treat the multi-label problem and topology cuts to keep topological constraints. Although the framework is general enough for any N-d image segmentation, we focus on tissue classification of 3-D brain images. Utilizing TOADS' topological template, we validated our algorithm with simulated images generated from the Brainweb phantom [8] and compared the results with TOADS. Segmentation results indicate our graph cuts approach improves running time compared with TOADS, with similar or better segmentations.

\section{Preliminaries}

\subsection{Graph cuts}

The two-terminal $(s-t)$ graph construction in a conventional graph cut scenario is as the following. Each pixel (voxel) in a 2-D (3-D) image is represented by a node in the graph. Other than these regular nodes, there are two special nodes called terminals. One terminal is the source $(s)$, usually standing for foreground, and the other terminal is the sink $(t)$ representing background. Each regular node is connected to the two terminals via two edges respectively, which are referred to as $t$-link. Neighboring regular nodes are connected by edges called $n$-link according to certain connectivity. Each edge is assigned a nonnegative weight, representing the flow capacity through this edge. The graph can be divided into two parts by deleting a set of edges so that each part contains one of the terminals. The cost of a cut is defined to be the sum of edge weights in the removed set.

Let $\mathcal{P}$ be the set of all non-terminal nodes. The labeling $f$ corresponding to a cut naturally defines a Markov Random Fields (MRF) type of energy

$$
E(f)=\sum_{p \in \mathcal{P}} D_{p}\left(f_{p}\right)+\sum_{(p, q) \in \mathcal{N}} V_{p, q}\left(f_{p}, f_{q}\right)
$$

where $f_{p}$ is the label for node $p$ and $\mathcal{N}$ is the local neighborhood, e.g., 8-neighborhood for 2-D images and 26neighborhood for 3-D. Function $D_{p}$ refers to the data term, which reflects the penalty of assigning node $p$ to label $f_{p}$. The more likely that $p$ has label $f_{p}$, the smaller the penalty should be. Function $V_{p, q}$ is the smooth term that penalizes discontinuities between neighboring nodes. Thus, the minimum of energy (1) corresponds naturally with the global optimum of a segmentation.

According to the max-flow/min-cut theory, the minimum cut in a $s-t$ graph equals the maximum flow from $s$ to $t$. There are many algorithms to find the max flow. Boykov and Kolmogorov proposed an efficient augmenting paths algorithm [5] based on searching two trees (rooted at $s$ and $t$ respectively) dynamically. In their algorithm, each node can have three states: active, passive or free. The active nodes are the outer boundary of the tree and therefore can grow. The passive nodes are blocked by nodes in the same tree. The algorithm iteratively performs the following processes: growth: grow two trees from $S$ and $T$ through nonsaturated edges until they touch giving a $s \rightarrow t$ path.

augmentation: augment the path with the maximum possible flow, the saturated edges break and trees turn into forests.

adoption: the isolated parts are connected to their original trees through non-saturated edges if possible; if not, set them to be free.

The algorithm ends when there is no active nodes and the segmentation is determined by the tree membership.

\subsection{Digital topology}

Now we discuss some basic definitions in digital topology for binary images. When considering topology on a pixel or voxel grid, one important concept is the connectivity, e.g., the commonly used connectivity for a 2-D image is 4 or 8 connectivity, and for the 3 -D case the 6,18 or 26 connectivity. The connectivity of an object and its complement cannot be arbitrary, and the valid foreground/background (or vice versa) pairs are $(4,8)$ for 2 -D and $(6,18),(6,26)$ for 3-D.

Although the topology is a global property, topology changes of an evolving object only take place at the nonsimple points on the boundary [9]. A simple point is a point whose change of labels does not change the number of connected components for both foreground and background in a local neighborhood system. Therefore, allowing label changes only for simple points will preserve the topology of an object. This simple point criterion has been used in levelset evolution [13], topology cuts, and TOADS.

\subsection{Topology preserving max-flow}

Here we briefly describe the topology preserving maxflow algorithm in [10], which is based on the BoykovKolmogorov algorithm [5]. Instead of two trees $S$ and $T$, Danek and Maska used four trees: $S_{F}, S_{B}, T_{F}$ and $T_{B} . F$ and $B$ are labels representing foreground and background initialized from the topological interface. The idea of using $F / B$ was first introduced by Zeng et al. [19].

The four trees are maintained dynamically as follows. A free node is recruited by a tree if it has the same $F / B$ label or it is simple; If two trees with the same terminal ( $S$ or $T)$ meet, points of $S_{B}\left(T_{F}\right)$ will be recruited by $S_{F}\left(T_{B}\right)$ if they are simple; If two trees with different terminals meet, a $s \rightarrow t$ path is found and augmented as in the BoykovKolmogorov algorithm, followed by the adoption process 
within each (sub) tree.

The segmentation result is determined not by the tree membership but by the $F / B$ labeling. Notice that label changes only occur at the boundary between foreground and background. To ensure homogeneous propagation, Danek and Maska store nodes according to a distance map so that active nodes closest to the frontier will be considered first. The distance map is updated whenever there is a node label change.

\section{4. $\alpha-\beta$ swap}

Boykov et al. [6] proposed two large moves: $\alpha$ expansion and $\alpha-\beta$ swap to fast approximate global energy minimum of (1) in case of multiple objects. Although $\alpha$ expansion is considered more accurate and efficient, we still choose $\alpha-\beta$ swap mainly based on two reasons. First, checking simple points is computationally expensive. Since $\alpha$ expansion considers all the labels in one graph cut, which means for even one node label change, it requires checking simple point criterion for all the objects to maintain their topology. On the other hand, $\alpha-\beta$ swap always checks simple points for only two labels, which is more straightforward. Although this may not be a major issue in this work, where a brain is classified into only three tissue classes, however, it will become critical if we extend our algorithm to classify more sub-structures of the brain. Second, compared with $\alpha$ expansion, $\alpha-\beta$ swap can be applied to more general energy functions and this may benefit us if we want to add shape priors into the energy function.

The key idea of $\alpha-\beta$ swap is to iteratively perform graph cuts between all pairs of labels with specific graph construction. In the graph cut of (say) $\alpha$ and $\beta$ labeled objects, the nodes of all other labels are kept fixed. Please refer to [6] for details. In our implementation, we adopt a more efficient graph setup for the $\alpha-\beta$ swap according to Kolmogorov and Zabih [15].

\section{Proposed Algorithm}

We use TOADS' topological template for initialization, and propose an iterative algorithm based on the $\alpha-\beta$ swap idea. In each iteration, graph cuts are performed for each pair of labels by calculating topology preserving max-flow. Special care is taken to guarantee that each object has the same topology as in the atlas. In our model, the data term in (1) is a function of intensity centroid, which is first learned from the initial template, and then updated after each iteration.

\subsection{Initialization}

The topology template we use is shown in Figure 1. It was created from combining a digital version of the Talairach atlas and the MNI/ICBM atlas [1], and editing it
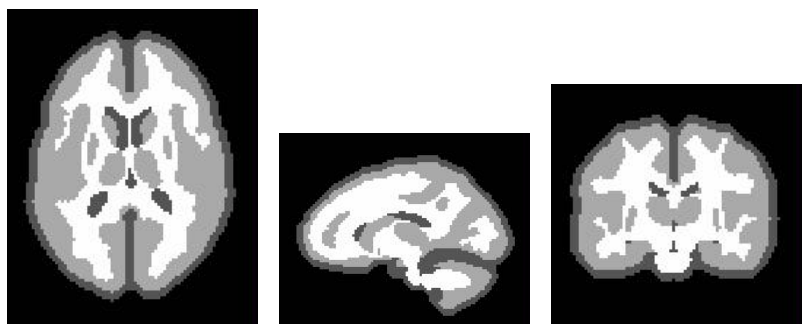

Figure 1. Topology template for the brain: axial, sagittal, and coronal views. White corresponds to white matter, light gray to gray matter, dark gray to CSF. Each tissue type includes several disconnected regions, e.g., CSF includes sulcal CSF and ventricles. Regions have the topology specified in Table 1.

through semiautomatic processing until reaching the desired topology for each structure. Although the template looks like an actual brain, the regions are very coarsely defined, since it is not expected to directly affect the geometry of the final segmentation. In this work, we are interested in segmenting brain images into three tissue classes: cerebrospinal fluid (CSF), grey matter, and white matter. Table 1 summarizes topology properties of the three structures. Similar to TOADS, we first register the template to the original image via rigid transformation, so that the template and image will overlap at the beginning phase.

For binary images, graph cuts has the advantage of low sensitivity to initialization, since it can minimize energy (1) globally as long as the energy is regular [15]. However, with topological constraints and multiple labels, the global optimum is not guaranteed. Moreover, as will be discussed next, we model the data function in terms of intensity centroid, which is learned from the initial interface. Therefore, the initialization is crucial to our problem. In this work, we run one iteration of TOADS from the registered template to ensure a better initialization. This process adds only a small overhead to the run-time of the whole algorithm.

\subsection{Graph construction}

Recall the energy function (1). Let $\mathcal{L}$ be the set of all labels and $\mathcal{P}\left(f_{p}\right)$ the set of points having label $f_{p}$. We define the data term to be

$$
\sum_{p \in \mathcal{P}} D_{p}\left(f_{p}\right)=\sum_{f_{p} \in \mathcal{L}} \sum_{p \in \mathcal{P}\left(f_{p}\right)} w_{p}\left(f_{p}\right)
$$

where

$$
w_{p}\left(f_{p}\right)=\lambda \cdot\left|c\left(f_{p}\right)-I_{p}\right|
$$

The coefficient $\lambda \geq 0$ specifies a relative importance of the data term versus the smooth term. $c\left(f_{p}\right)$ is the intensity centroid for label $f_{p}$ and is defined as $\sum_{p \in \mathcal{P}\left(f_{p}\right)} I_{p} / \sharp \mathcal{P}\left(f_{p}\right)$, where $\sharp \mathcal{P}\left(f_{p}\right)$ is the number of voxels in $\mathcal{P}\left(f_{p}\right)$ and $I_{p}$ is the intensity value at voxel $p$. The function in (3) is reasonable since voxel $p$ is more likely to be assigned with label 


\begin{tabular}{|c|c|c|c|c|}
\hline Tissue & Euler & Connected & Internal & Topological \\
CSF & 4 & 3 & 1 & 2 \\
Characteristic & Components & Cavities & 1 \\
Grey Matter & 6 & 4 & 0 & 7 \\
Whiter Matter & -8 & 1 & 2 & 7 \\
\hline
\end{tabular}

Table 1. Topological properties of the brain template.

$f_{p}$ if the intensity difference is small. The centroid $c\left(f_{p}\right)$ is learned from the initial topology interface and updated iteratively during the algorithm. The choice of using $L 1$ norm instead of the $L 2$ norm is based on experimental results.

The smooth term in (1) is defined as

$$
\sum_{(p, q) \in \mathcal{N}} V_{p, q}\left(f_{p}, f_{q}\right)=\sum_{(p, q) \in \mathcal{N}} w_{(p, q)} \cdot \delta\left(f_{p}, f_{q}\right)
$$

where

$$
\delta\left(f_{p}, f_{q}\right)= \begin{cases}1 & \text { if } f_{p} \neq f_{q} \\ 0 & \text { otherwise }\end{cases}
$$

and

$$
w_{(p, q)}=\exp \left(-\frac{\left(I_{p}-I_{q}\right)^{2}}{2 \sigma^{2}}\right) \cdot \frac{1}{\|p-q\|}
$$

The delta function (5) makes the smooth function only account for discontinuities, i.e., when the labels of two neighboring nodes are different. $\sigma$ is a parameter and function (6) penalizes a lot when $\left|I_{p}-I_{q}\right| \leq \sigma$, indicating that it will be unlikely to cut an edge between two nodes having similar intensities. $\|p-q\|$ is the Euclidean distance between two voxels in a 26-neighborhood system.

In each iteration of our algorithm, we perform one graph cut for each pair of labels, and the graph is constructed using the method in [15]. $n$-link cost is set to be $w_{(p, q)}$, and $t$-link cost is set to be $w_{p}\left(\bar{f}_{p}\right)$, where $\bar{f}_{p}$ is the opposite label of $f_{p}$ (for each pair, there is only two labels, i.e., 0 and 1 ).

\subsection{Topological issues}

As mentioned in Section 2.2, the connectivity of foreground and background must be compatible. However, in the case of multiple labels, the connectivity must be kept the same for each label. This is because one object may be the foreground in a graph cut of a particular pair of labels, but be set as background for the graph cut of another pair. Different connectivity will create isolated parts which violates the topological constraints. In this work, we set the connectivity to be 6 for all objects in the image. Higher connectivity 18 or 26 may increase segmentation accuracy, however, it may cause the problem of mutual intersection, a situation where two objects connect through the same point (for instance, the black and white squares of a checkerboard would all mutually intersect in 18 or 26 connectivity). Please refer to [2] for a detailed discussion on the topological issues for multiple objects.
Recall that in Section 2.3, Danek and Maska used a distance map to keep track of the boundary between foreground and background. Here we use two distance maps instead of one. Consider our multi-label problem, when performing an $\alpha-\beta$ swap for two labels, say $l_{1}$ and $l_{2}$, we are actually dealing with three objects: $O_{l_{1}}, O_{l_{2}}$ and $O_{b}$, where $b$ is the union of all labels other than $l_{1}$ and $l_{2}$. Although label of nodes in $O_{b}$ are fixed, when checking simple points on the boundary of $O_{l_{1}}$ or $O_{l_{2}}$, the nodes in $O_{b}$ close to the frontier will get involved, since they are in the neighborhood of some other points inside $O_{l_{1}}$ or $O_{l_{2}}$. Hence, we use two distance maps. One map sets $O_{l_{1}}$ to be foreground and nodes of all other labels to be background, vice versa for the other map. The two maps are updated whenever there is a label change. The simple point criterion on both maps should be satisfied to determine whether a point is simple. To check the simple point criterion for 3-D connectivity, we use the technique in [16].

\subsection{Algorithm}

The complete algorithm is summarized as follows:

1. Register the topological template to the image.

2. Perform one iteration of TOADS based on the registered template, set initial labeling $f$ according to the resulting segmentation.

3. Calculate intensity centroids.

4. For each pair of labels $(\alpha, \beta) \in \mathcal{L}$, perform one $\alpha-\beta$ swap with topology preserving max-flow.

5. If the convergence criterion is not satisfied, loop to step 3.

\section{Return $f$.}

The convergence criterion is the relative change of intensity centroid for each label being less than $1 \%$. The algorithm is implemented using $\mathrm{C}++$ with a MATLAB interface, based on a graph cut library created by the Center for Biomedical Analysis (http://cbia.fi.muni.cz/projects/graphcut-library.html). 

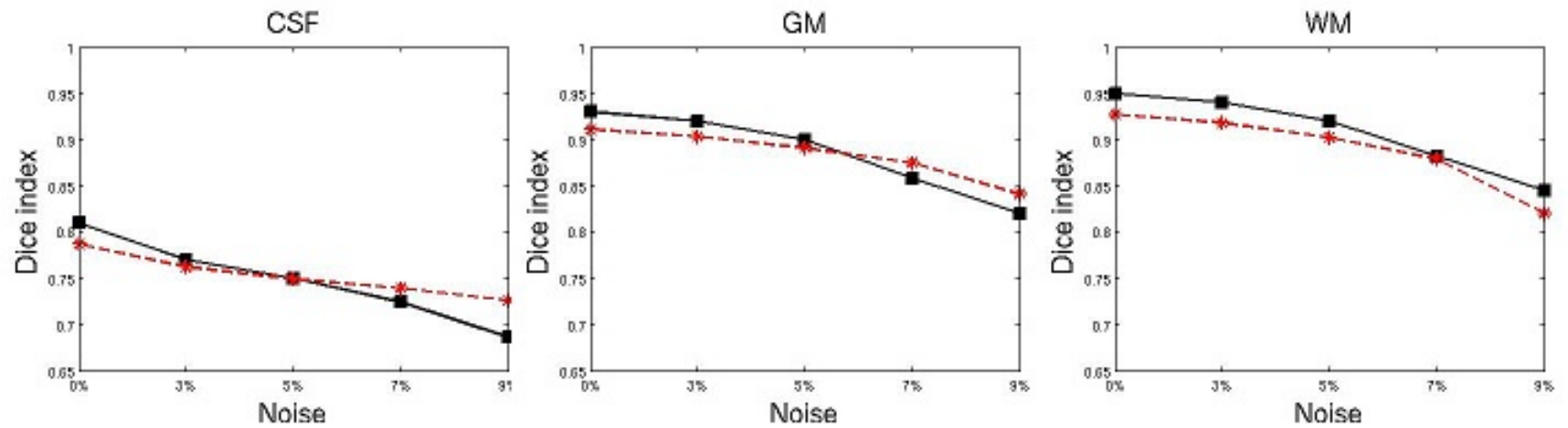

Figure 2. Dice index for the segmentation of Brainweb phantom images, red dashed asterisk line for TOADS and black solid square line for graph cuts.

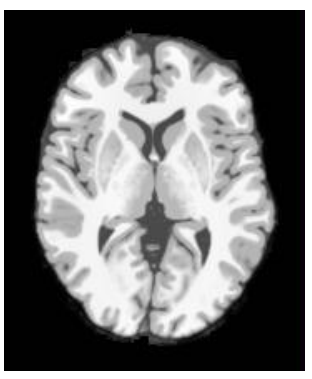

(a)

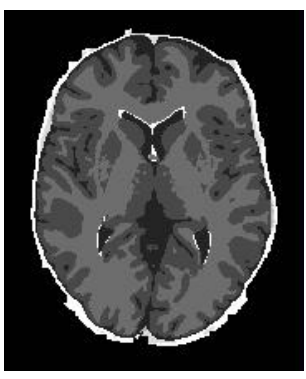

(b)

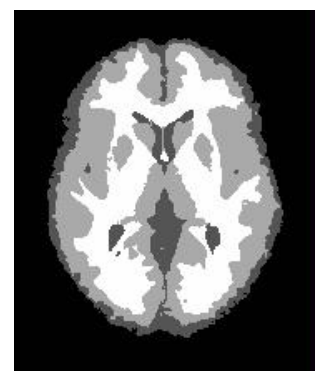

(c)

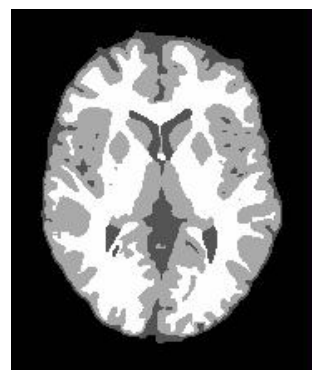

(d)

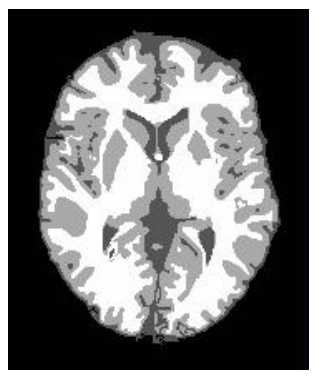

(e)

Figure 3. Segmentation example of TOADS and graph cuts. (a): original image from Brainweb phantom without noise; (b): ground truth segmentation; (c): Registered brain template followed by one iteration of TOAD, used as the initialization of the graph cuts algorithm; (d): TOADS segmentation; (e): segmentation using the graph cuts method.

\section{Experiments}

We perform several validation experiments to compare our results with TOADS using simulated images generated from the Brainweb phantom [8]. The images are created as $1 \mathrm{~mm}$ T1 images, with $181 \times 217 \times 181$ voxels and increasing levels of noise from $0 \%$ to $9 \%$. TOADS can be downloaded from (http://www.nitrc.org/projects/toads-cruise/), and was run using default parameters. We use 26-neighborhood system in our graph cuts algorithm with a unique set of parameters for all images. Both TOADS and the graph cuts algorithm use a topological template with four objects: background, cerebro-spinal fluid (CSF), grey matter (GM) and white matter (WM). The images were first processed to remove extra-cerebral tissues using SPECTRE [7], a skull stripping technique.

The overlap between the segmentations and the Brainweb ground truth was computed using the Dice overlap coefficient $(D(X, Y)=2|X \cap Y| /(|X|+|Y|))$ for white matter, gray matter and CSF respectively, which are shown in Figure 2. The Brainweb ground truth has arbitrary topology and therefore TOADS and our results will always deviate from it because the latter two are constrained to preserve

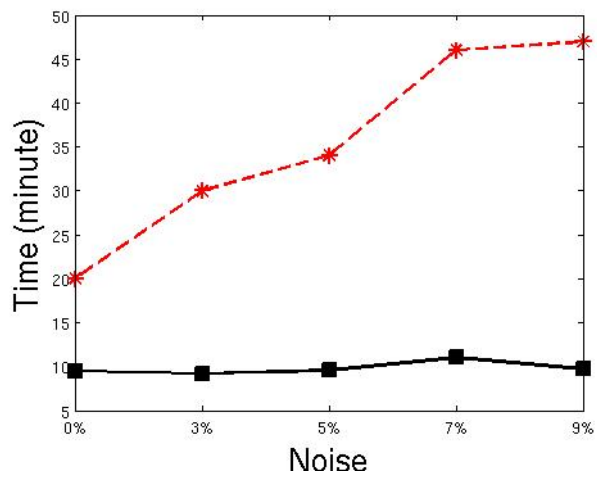

Figure 4. Running time for the segmentation of Brainweb phantom images, red dashed asterisk line for TOADS and black solid square line for graph cuts.

topology as in Table 1. Figure 2 indicates that our segmentation is more accurate when the noise level is low (less than 5\%), and TOADS does better for higher noise levels (for CSF and GM). An example of TOADS and our segmentation for an image without noise is given in Figure 3. 
The Brainweb image serving as ground truth is a anatomical model consisting of a set of 9 "fuzz"tissue membership volumes, including white matter, grey matter, cerebrospinal fluid and others. Figure 3 (b) shows a view of the ground truth image after the skull stripping pre-process, which is used in our Dice calculation. There is clearly other labels, e.g., skull and skin (on the outer boundary of CSF) and glial matter (on the boundary of the ventricles). The remaining skull and skin tissues reflect errors in the skull stripping and therefore cause the Dice results for TOADS and our algorithm to be low for CSF (Figure 2). The reason why we keep all other labels in the ground truth is because it is a scenario more similar to practical cases, where one may not expect perfect skull stripping.

Since one of our motivations to use graph cuts is its computational efficiency, we compare running time of the two algorithms, which is shown in Figure 4. All experiments are performed on a AMD Opteron $2.73 \mathrm{Ghz}$ PC. The results show that our algorithm is more efficient and independent of the amount of noise in the image, whereas TOADS converges more slowly for larger noise levels.

\section{Conclusions}

In this paper, we proposed a multi-label image segmentation framework which respects topology. The framework originates from another topology preserving segmentation technique (TOADS), and incorporates advantages of graph cuts, a method which has proven to be efficient for a variety of segmentation problems in computer vision. The framework is validated by extracting cerebro-spinal fluid, grey matter and white matter from simulated brain magnetic resonance images. The results show that our algorithm is about three times more efficient on average than TOADS, with similar or better segmentations.

In the future, topological constraints of groups of objects [3] can be embedded into our graph cuts framework. We may also consider including statistical atlases by introducing shape priors to the graph construction, e.g., as in [17]. These attempts will enable our framework to segment subcortical structures with similar intensities, such as the hippocampus and amygdala.

\section{References}

[1] P.-L. Bazin, M. McAuliffe, W. Gandler, and D. Pham. Free software tools for atlas-based volumetric neuroimage analysis. SPIE Med. Img. Conf., San Diego, CA, 2005. 187

[2] P.-L. Bazin and D. Pham. Topology-preserving tissue classification of magnetic resonance brain images. IEEE Transactions on Medical Imaging, 26(4):487-496, 2007. 185, 188

[3] P.-L. Bazin and D. Pham. Homeomorphic brain image segmentation with topological and statistical atlases. Medical Image Analysis, 12(5):616-625, 2008. 185, 190
[4] Y. Boykov and G. Funka-lea. Graph cuts and efficient n$\mathrm{d}$ image segmentation. International Journal of Computer Vision, 70(2):109-131, 2006. 185

[5] Y. Boykov and V. Kolmogorov. An experimental comparison of min-cut/max-flow algorithms for energy minimization in vision. IEEE Transactions on Pattern Analysis and Machine Intelligence, 26(9):1124-1137, 2004. 185, 186

[6] Y. Boykov, O. Veksler, and R. Zabih. Fast approximate energy minimization via graph cuts. IEEE Transactions on Pattern Analysis and Machine Intelligence, 23(11):1222-1239, 2001. 185, 187

[7] A. Carass, J. Cuzzocreo, M. Wheeler, P.-L. Bazin, S. Resnick, and J. Prince. Simple paradigm for extracerebral tissue removal: Algorithm and analysis. NeuroImage, 56(4):1982-1992, 2011. 189

[8] D. Collins, A. Zijdenbos, V. Kollokian, J. Sled, N. Kabani, C. Holmes, and A. Evans. Design and construction of a realistic digital brain phantom. IEEE Transactions on Medical Imaging, 17(3):463-468, 1998. 186, 189

[9] M. Couprie, F. Bezerra, and G. Bertrand. Topological operators for grayscale image processing. J. Electron. Imag., 10(4):1003-1015, 2001. 186

[10] O. Danek and M. Maska. A simple topology preserving max-flow algorithm for graph cut based image segmentation. Sixth Doctoral Workshop on Mathematical and Engineering Methods in Computer Science (MEMICS'10), 2010. 185, 186

[11] A. Delong and Y. Boykov. Globally optimal segmentation of multi-region objects. International Conference on Computer Vision (ICCV), 2009. 185

[12] D. Greig, B. Porteous, and A. Seheult. Exact maximum a posteriori estimation for binary images. Journal of the Royal Statistical Society, Series B, 51(2):487-496, 1989. 185

[13] X. Han, C. Xu, and J. L. Prince. A topology preserving level set method for geometric deformable models. IEEE Trans. Pattern Anal. Mach. Intell., 25(6):755-768, 2003. 186

[14] D. Hower, V. Singh, and S. Johnson. Label set perturbation for mrf based neuroimaging segmentation. International Conference on Computer Vision (ICCV), 2009. 185

[15] V. Kolmogorov and R. Zabih. What energy functions can be minimized via graph cuts? IEEE Trans. Pattern Anal. Mach. Intell., 26(2), 2004. 187, 188

[16] G. Malandain and G. Bertrand. Fast characterization of 3d simple points. 11th International Conference on Pattern Recognition, pages 232-235, 1992. 188

[17] J. Malcolm, Y. Rathi, and A. Tannenbaum. Graph cut segmentation with nonlinear shape priors. International Conference on Image Processing (ICIP), 2007. 190

[18] N. Vu and B. Manjunath. Shape prior segmentation of multiple objects with graph cuts. IEEE Conference on Computer Vision and Pattern Recognition (CVPR), 2008. 185

[19] Y. Zeng, D. Samaras, W. Chen, and Q. Peng. Topology cuts: A novel min-cut/max-flow algorithm for topology preserving segmentation in $\mathrm{n}-\mathrm{d}$ images. Computer Vision and Image Understanding, 112(1):81-90, 2008. 185, 186 